\title{
Systematic review of the changes in the microbiome following spinal cord injury: animal and human evidence
}

\author{
Ezra Valido $\mathbb{D}^{1,2}$, Alessandro Bertolo ${ }^{1,3}$, Gion Philip Fränkl $\mathbb{D}^{1,4}$, Oche Adam Itodo ${ }^{1,5}$, Tainá Pinheiro ${ }^{1,2}$, Jürgen Pannek (iD ${ }^{6,7}$, \\ Doris Kopp-Heim (iD) ${ }^{5,8}$, Marija Glisic (D) ${ }^{1,5}$ and Jivko Stoyanov (iD ${ }^{1,5}$ \\ (c) The Author(s) 2022
}

\begin{abstract}
STUDY DESIGN: Systematic review.
OBJECTIVES: To investigate the changes in the microbiome among human and animal populations with spinal cord injury (SCI). METHODS: Four databases (EMBASE, Medline (Ovid), Web of Science, Cochrane Central Register of Trials (CENTRAL)) and Google Scholar were searched. No language restrictions were applied. Data extraction was done in parallel and independently by two reviewers. The search was last conducted on 07 April 2021.

RESULTS: There were 6869 studies retrieved, 43 full-text studies reviewed, and 19 studies included. There were seven animal gut studies, six human gut studies, and six urinary tract studies identified. There were no publications found on other body sites. Among the included studies, we observed a consistent and significant difference in gut microbiome composition between populations with $\mathrm{SCl}$ and able-bodied populations. This is characterized by a decrease in beneficial butyrate-producing bacteria (Faecalbacterium, Megamonas, Roseburia) and an increase in inflammation-associated bacteria (Alistipes, Anaerotruncus, and Lachnoclostridium). On the other hand, the urine of individuals with $\mathrm{SCl}$ was polymicrobial and members of Enterobacteriaceae (Escherichia coli, Klebsiella pneumoniae) were frequently observed. Probiotics were shown to induce a significant but transient shift in the urinary tract microbiome. The studies had low to moderate risks of bias.

CONCLUSIONS: There are limited studies on the changes in microbiome among $\mathrm{SCl}$ populations. The gut microbiome was characterized by bacterial profiles associated with chronic inflammation and metabolic disorder while the studies of the urinary tract microbiome show the dominance of bacterial genera associated with urinary tract infection.
\end{abstract}

Spinal Cord (2022) 60:288-300; https://doi.org/10.1038/s41393-021-00737-y

\section{INTRODUCTION}

After spinal cord injury (SCl), the loss of innervation on organ systems below the level of lesion induces changes that affect basic body functions. This disturbs the gut and urinary tract motility, glandular secretions, and the overall tone of vascular vessels, muscles, and skin. These changes influence the availability and distribution of nutrients and metabolites needed for the growth of indigenous microbial communities. Depletion or enrichment of microorganisms at an organ-system level is called dysbiosis and can lead to higher risks of infections and increased risks of chronic illnesses such as metabolic disorders, cardiovascular-related morbidities, and autoimmune diseases [1]. Thus, investigating the changes in the microbial communities can impact clinical decisions for individuals with $\mathrm{SCl}$.

Traditionally, the investigation of dysbiosis is through microflora studies via microbial culture methods. The developments in genome sequencing allowed better microbial characterization and identification by studying microbiomes or the collective genome of microorganisms in a specific organ or location at a certain time point [2]. This technique identified previously undetected species and provided evidence of microbial presence on body sites thought to be sterile. Microbiome studies have increased steadily but remained focused on able-bodied individuals with diseases. Growing evidence of specific organisms associated with illnesses is changing medical therapy and therefore understanding the changes in the microbiomes of individuals with $\mathrm{SCl}$ can improve medical therapies in this population.

There are narrative reviews regarding gut microbiomes among individuals with $\mathrm{SCl}[1,3,4]$. The focus of these reviews is on the inter-relationship of the physiological changes after injury, the immune response, and how the microbiome has changed. These reviews are not systematic and did not explore systematically the bacterial taxa that are enriched or depleted in the microbiome of individuals with $\mathrm{SCl}$. Moreover, to our knowledge, there are no reviews summarizing the evidence on the other body locations in this population. Thus, this systemic review aims to provide a comprehensive overview of the literature concerning the changes in the microbiome in humans and animals with $\mathrm{SCl}$, identify literature gaps and critically appraise the quality of the existing evidence to provide directions for future research.

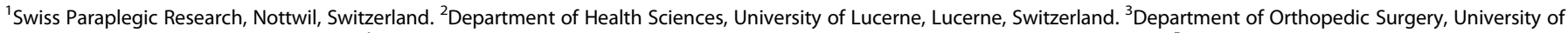

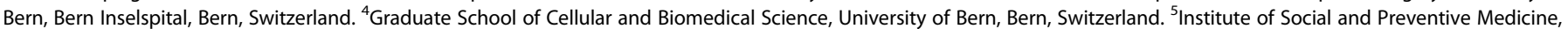

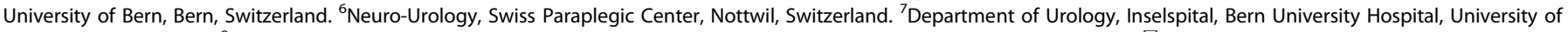
Bern, Bern, Switzerland. ${ }^{8}$ Public Health \& Primary Care Library, University Library of Bern, University of Bern, Bern, Switzerland. ${ }^{凶}$ email: ezra.valido@paraplegie.ch
} 


\section{METHODS}

Data sources and search strategy

This review was conducted in accordance with Preferred Reporting Items for Systematic Reviews and Meta-Analyses [5] and the steps described by Muka et al. [6]. The search strategy was created by an experienced information specialist using terms related to $\mathrm{SCl}$ such as paraplegia, tetraplegia, and nontraumatic causes such as spina bifida. These terms were combined with terms for microbiome, microbiota and terms related to their changes such as dysbiosis. Details of the search strategy are provided in Supplementary A. Four electronic databases were searched (EMBASE, Medline (Ovid), Web of Science, the Cochrane Central Register of Controlled Trials (CENTRAL)) and Google Scholar from inception to 07 April 2021 without language restrictions. Detailed study protocol can be found in PROSPERO (CRD42020185555).

\section{Study selection and eligibility criteria}

Studies were included with the following criteria: (1) animal, observational and clinical studies (2) reported the microbial community characteristics through the microbiome via genome or $16 \mathrm{~S}$ ribosomal ribonucleic acid (16S rRNA) gene sequencing (3) included a population of persons or animals with $\mathrm{SCl}$. Excluded were conference abstracts, reviews, letter to editors, case studies, and non-peer-reviewed studies. Two reviewers independently screened the titles and abstracts for inclusion and assessed eligibility in their full text. Discrepancies were resolved by consensus and if necessary, a third reviewer was consulted.

\section{Data extraction}

Two reviewers independently extracted data using a predesigned table that includes the primary author and year, study characteristics, sequencing technique, microbiome characteristics, diversity indices reported, and other relevant findings. Diversity indices included $\alpha$ and $\beta$ diversity indices of the microbiome. The a diversity (Chao1, Species or Operational Taxonomic Unit [OTU] or Amplicon Sequence Variant [ASV] counts, ACE, Shannon, Simpson, Inverse Simpson, phylogenetic diversity and Fischer a) measures the diversity within a group while the $\beta$ diversity (Bray-Curtis, weighted/unweighted UniFrac) measures the diversity between groups [7]. The Human Microbiome Project consortium defines diversity as the number and abundance distribution of specific organisms [8]. The depletion or enrichment of the members of the microbiome were identified as well.

\section{Assessing the risk of bias}

The risk assessment tool used was the Office of Health Assessment \& Translation (OHAT) risk of bias tool by the US Department of Health and Human Services [9]. Risk assessment was based on study type. Classification was set with questions on selection, confounding, performance, attrition, and detection biases. Lowrisk studies had no probable or definitely high risk or no insufficient information. Moderate-risk studies had probable high risks or insufficient information in any of the biases but without definitely high risk. High-risk studies had a definitely high-risk assessment from one of the biases. Two reviewers independently assessed each study and rated the studies according to OHAT protocol. Discrepancies in risk assessment were resolved by consensus and if needed, consultation with a third reviewer.

\section{RESULTS}

\section{Characteristics of included studies}

There were 10,329 studies identified. From these, 6869 unique titles and abstracts were screened, 43 full-text studies reviewed of which 19 studies met the inclusion criteria (Fig. 1). Thirteen studies reported on the gut microbiome and six on the urinary tract microbiome. Seven of the studies were animal studies, 12 were human studies with four with interventions and the remaining nine were observational studies. No studies were found on other organ-systems. All studies used the hypervariable regions of bacterial 16S rRNA as sequencing target.

\section{The gastrointestinal microbiome in animal studies of spinal cord injury}

We identified seven rodent $\mathrm{SCl}$ studies, four were conducted in mice and three in rats. Five studies included only females. Five studies induced $\mathrm{SCl}$ at thoracic (T9/T10) and two at cervical (C5) level. Six studies had intervention in their setting. Two of the studies had a pre-injury baseline of the gut microbial composition. Summarized findings of the microbiome changes can be found in Table 1. Five of the seven animal studies had a low risk of bias. Two had moderate risk and used antibiotics post-injury that could have affected the detected microbiomes [10, 11] (Supplementary Table 1).

The animal studies showed changes in microbial composition after $\mathrm{SCl}$ but not necessarily in a diversity. O'Connor et al. [10] compared groups of female rats injured at T10 vs sham-operated rats with the use of gentamicin post-operation and found no significant difference in the Shannon diversity index. There was however significant difference in $\beta$ diversity at family, genus, and species levels between groups. Jing et al. [11] on the other hand, studied adult female mice with T10 injury vs sham injured mice and compared them to groups with fecal matter transplant (FMT) (SCI-FMT vs sham-FMT). There were significant changes in ACE and Chao diversity indices between $\mathrm{SCl}$ mice vs sham injured mice but no difference between SCI-FMT vs the sham group. Blautia, Anaerostipes, Lachnospiraceae NK4A136, Christensenellaceae were seen to significantly decrease in the $\mathrm{SCl}$ group but were alleviated by FMT. They conclude that FMT significantly influences the microbial community of the gut post-SCI [11]. Jing et al. [12] likewise investigated the use of melatonin $(10 \mathrm{mg} / \mathrm{kg}$, twice daily) in female mice with injury at T10. Melatonin led to significantly decreased $\mathrm{SCl}$-induced gut permeability and a decrease in microbial diversity and richness in SCl mice. Schmidt et al. [13] studied a group of female rats with cervical level $\mathrm{SCl}$. There were major differences in composition between the groups of $\mathrm{SCl}$ rats vs non-SCl rats and $\mathrm{SCl}$ rats vs $\mathrm{SCl}-\mathrm{FMT}$ rats but not in $\mathrm{SCl}-\mathrm{FMT}$ rats vs non-SCl rats 3 days post-SCl. The SCI-FMT group had reduced total numbers of altered genus-species OTU suggesting prevention of $\mathrm{SCl}$-induced dysbiosis. By 4 weeks post-SCl, the genusspecies OTU difference was reduced compared to pre-injury baseline indicating normalization of the microbiota composition. This is supported by the Shannon diversity index with increased in all groups 3 days post-SCI but were all similar after 4 weeks post$\mathrm{SCl}$ when compared to pre-injury $\beta$ diversity at the genus-species level indicates proximity in the microbial composition of the groups of $\mathrm{SCl}-\mathrm{FMT}$ rat and non-SCl group indicating similarity. Schmidt et al. [14] subsequently investigated with FMT from anxious rat donors and confirmed previous data on $\mathrm{SCl}$-induced dysbiosis but there was no difference in $\beta$ diversity and reduced amount of Lactobacillus between SCl rats vs SCI-FMT. This indicates that successful FMT depends on the donors' microbiome as well [14]. In another study, Myers et al. [15] studied the influence of gram-negative derived lipopolysaccharides on inflammation by provoking endotoxin-mediated cyclic adenosine monophosphate specific Pde4 subfamily b (Pde4b) enzyme induced inflammation in gut dysbiosis post-SCl in female wild type (WT) mice and to mice without the enzyme. The authors reported a 2.5 -fold increase in $16 \mathrm{~S}$ rRNA gene copy in SCI WT mice compared to sham injured WT mice indicating bacterial enrichment post-SCl. The peak expression of inflammatory markers preceded the significant rise of systemic endotoxemia. Kigerl et al. [16] investigated whether SCl can cause bacterial translocation, the passage of viable bacteria from the gastrointestinal tract to extra-intestinal sites, and changes in gut permeability in mice. They confirmed the bacterial translocation by positive microbial 


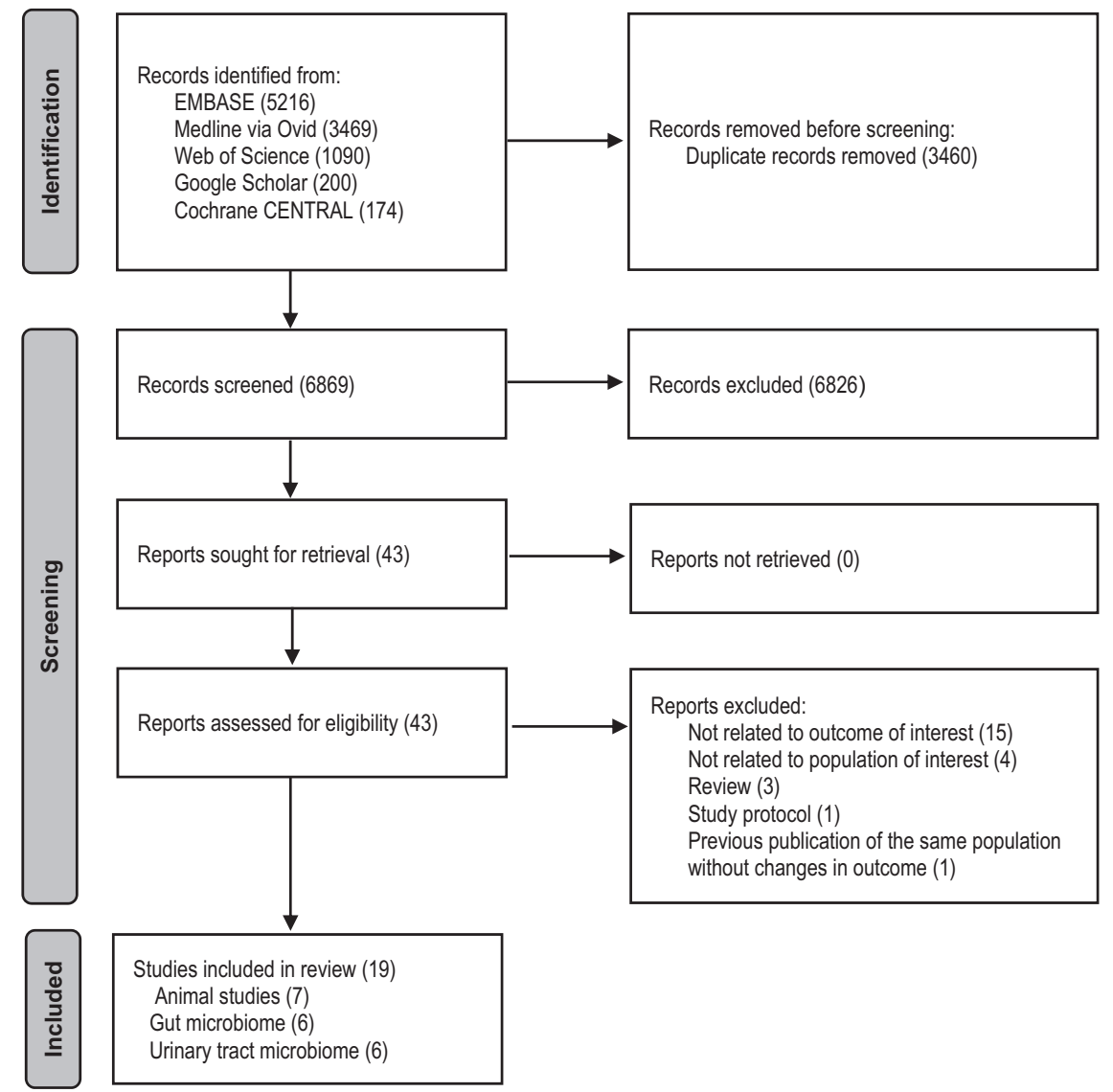

Fig. 1 Flowchart of study inclusion. PRISMA flow diagram for the changes in the microbiome following spinal cord injury resulting from searches of databases, screening, reasons for exclusion and description of included studies.

culture of the liver, spleen, kidneys, mesentery, and blood in injured mice and none were found in non-SCl mice after 7 days. This was supported by a $20 \%$ increase in gut permeability seen in the leakage of fluorescein isothiocyanate-dextran (4kD) when compared to the blood FICT fluorescence $4 \mathrm{~h}$ after lavage in $\mathrm{SCl}-$ mice 7 days post-SCl. They further showed that the gut-associated lymphoid tissue (GALT) in mice was activated. Kigerl et al. used VSL\#3 probiotic in mice, comprising Lactobacillus casei, Lactobacillus plantarum, Lactobacillus acidophilus, Lactobacillus debrueckii subsp bulgaricus, Bifidobacterium longum, Bifidobacterium breve, Bifidobacterium infantis and Streptococcus salivaris subsp thermophiles, from the day of injury to 35 days post-SCI in mice and showed improved functional recovery, reduced spinal lesion and significant transient gut microbiome enrichment of Bifidobacteriales and Lactobacillales.

\section{Spinal cord injury and gastrointestinal tract microbiome in humans}

Five case-control studies of persons with chronic traumatic $\mathrm{SCl}$ (more than 6 months) and one interventional study with spina bifida compared to healthy able-bodied individuals were included. The studies of Zhang et al. [17, 18] were grouped as they contain the same population. Two studies were assessed with a low risk of bias and four with moderate risks (Supplementary Table 1). Two studies exclusively studied male individuals $[17,18]$ and two studies lack the description of the extent of injury $[19,20]$. One study has a subgroup of acutely injured $\mathrm{SCl}$ wherein the individuals had antibiotics for their health management [21] and another included incomplete SCI [19]. All studies targeted 16S rRNA variable regions or microbiome analysis. Summarized findings of the microbiome changes are in Table 2.
Five of the studies included adult populations with two studies subdividing their study population by level of SCl. The Zhang et al. $[17,18]$ studies recruited only male individuals with chronic thoracolumbar $\mathrm{SCl}$ (TL-SCl) or cervical-SCl (C-SCl) and compared them to healthy able-bodied individuals. The TL-SCI group had significantly higher Chao1 and lower Simpson index vs ablebodied individuals. There was a significant difference in $\beta$ diversity at the phylum level across the three groups. Among the top OTUs Bacteroides and Blautia were increased in $\mathrm{SCl}$ while Prevotella, and [Eubacterium] rectale showed a significant decrease in $\mathrm{SCl}$ and Faecalbacterium decreased only in C-SCI $[17,18]$. Significant serum level correlations of high-density lipoprotein (HDL) (negative) and apoA1 (positive) with the changes at microbial genus levels. Lowdensity lipoprotein and glucose positively correlated with changes at phylum level in C-SCl. Additionally, the team noted that the presence of Bacteroides and Blautia were significantly associated with lipid metabolism in C-SCl than in able-bodied individuals and Faecalbacterium, Megamonas and Prevotella correlated negatively with lipid metabolism biomarkers while Lactobacillus correlated positively to glucose [17]. In contrast, Gungor et al. [22] classified their study based on the bowel dysfunctional manifestation of individuals with $\mathrm{SCl}$ and only included individuals with $\mathrm{SCl}$ above T6 or cauda equina syndrome. They found that the gut microbiome in $\mathrm{SCl}$ was dominated by Blautia, Bifidobacterium, Faecalbacterium, and Ruminococcus and between groups, Roseburia, Pseudobutyrivibrio, Dialister, Marvinbryantia, and Megamonas were significantly different. The authors concluded that there was a significant reduction of butyrate-producing bacteria among individuals with $\mathrm{SCl}$.

Lin et al. [19] recruited individuals with chronic traumatic $\mathrm{SCl}$ but only $22 \%$ had complete $\mathrm{SCl}$. The extent of loss of bowel 

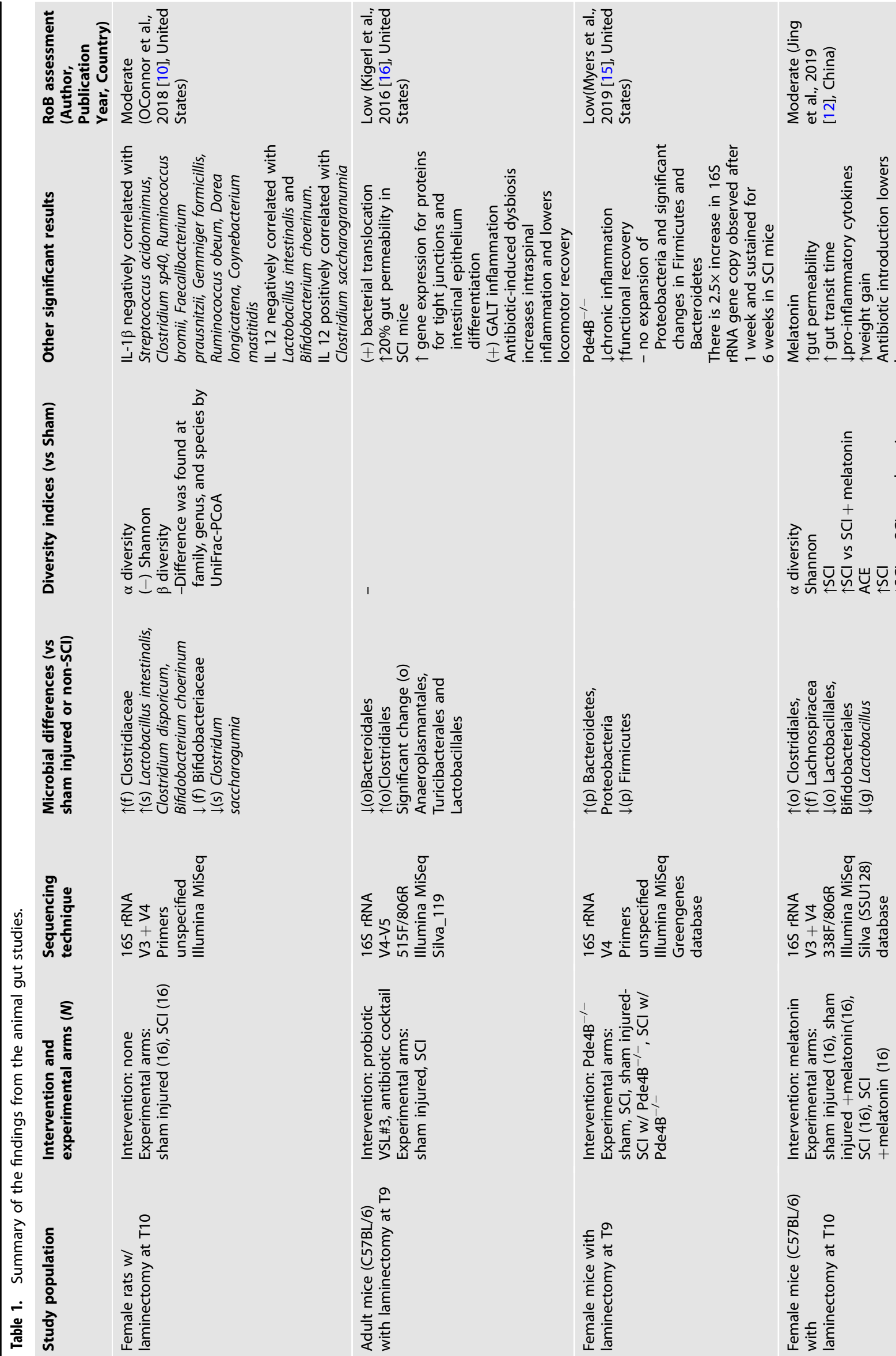

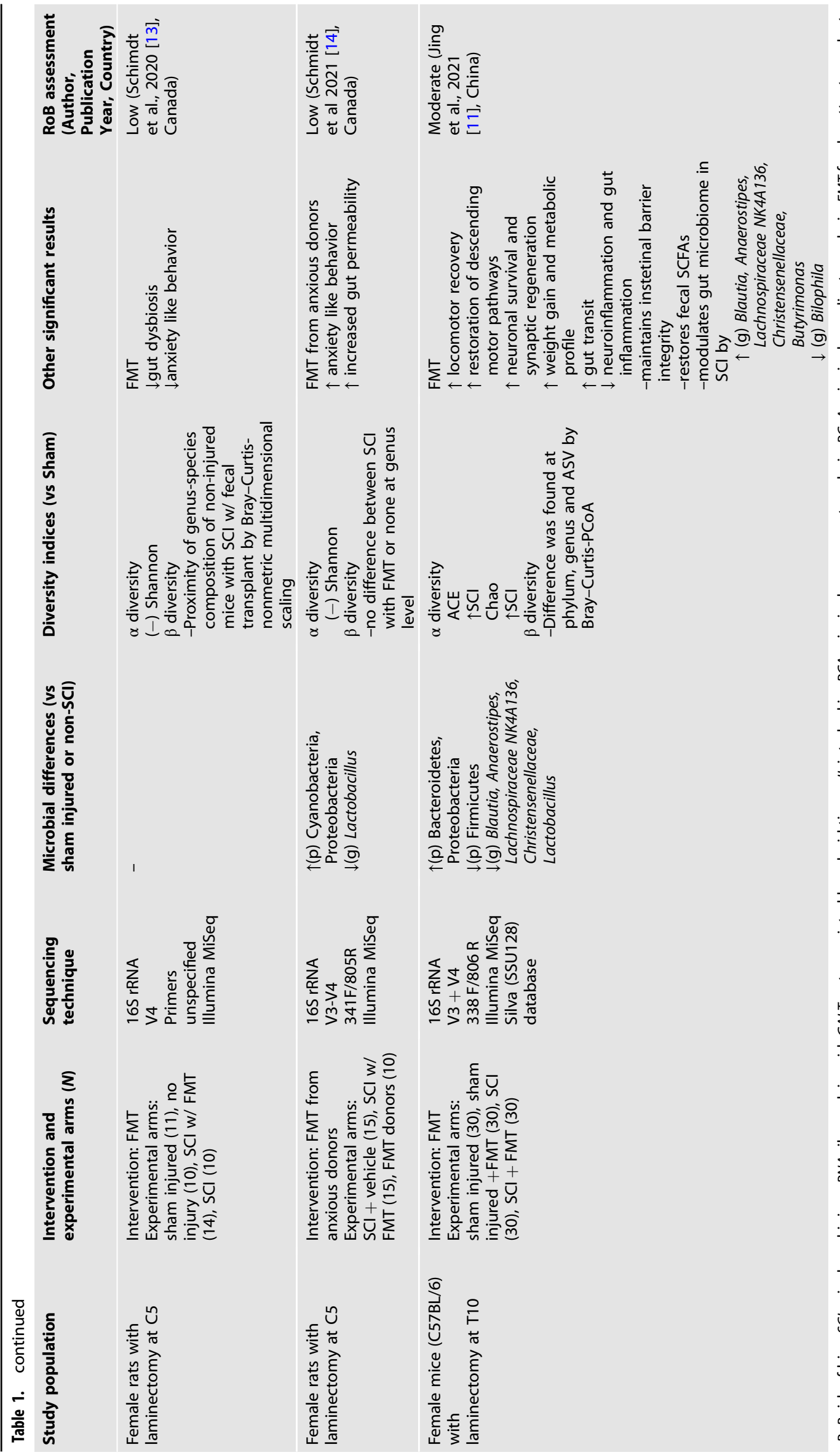

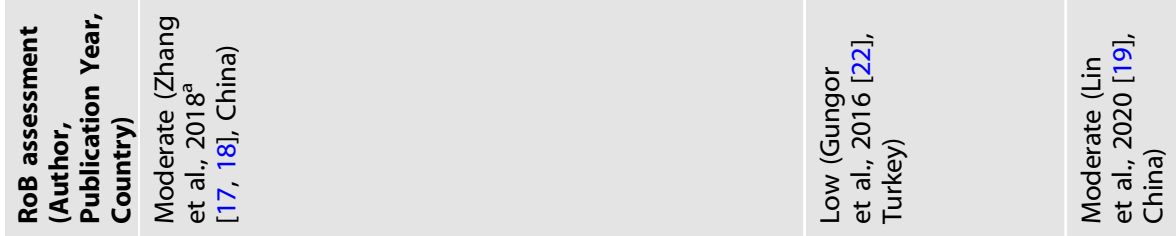

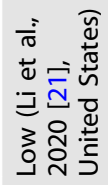
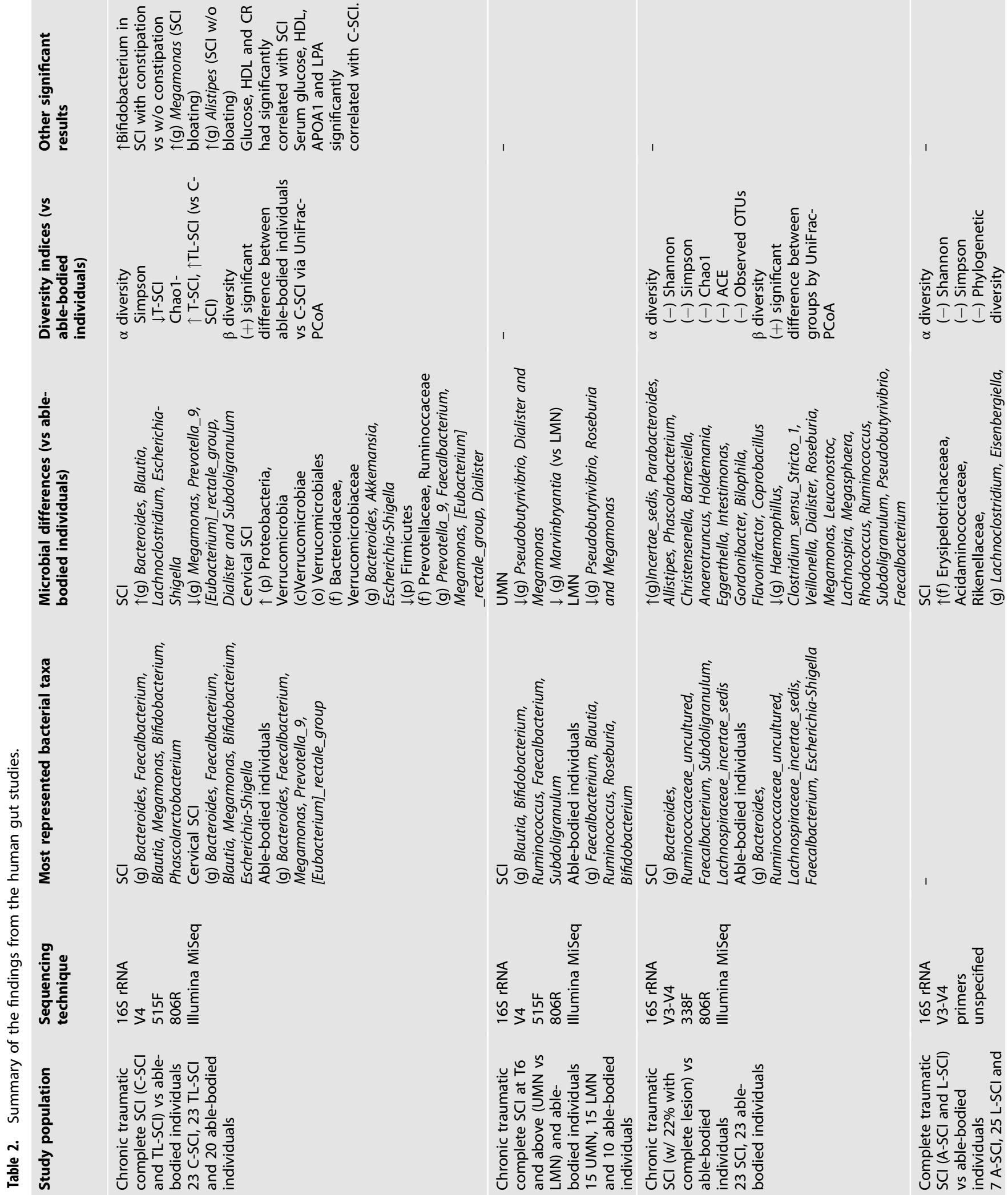

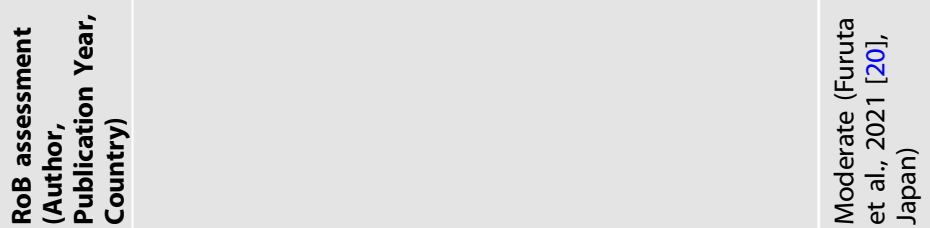

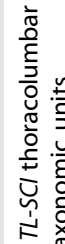
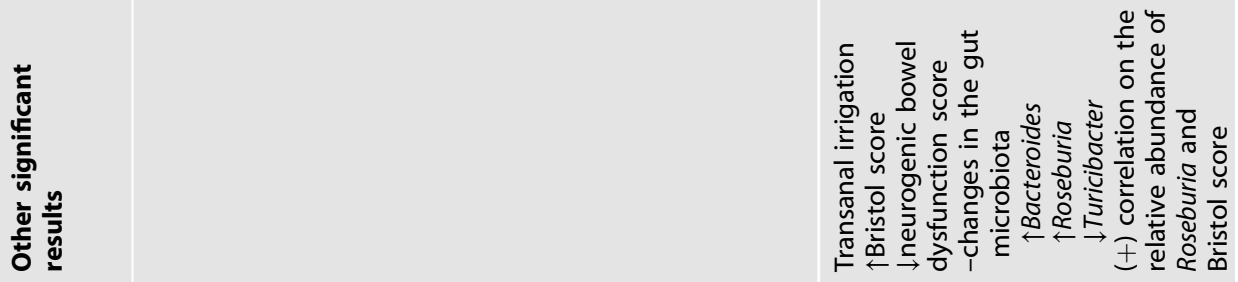
这
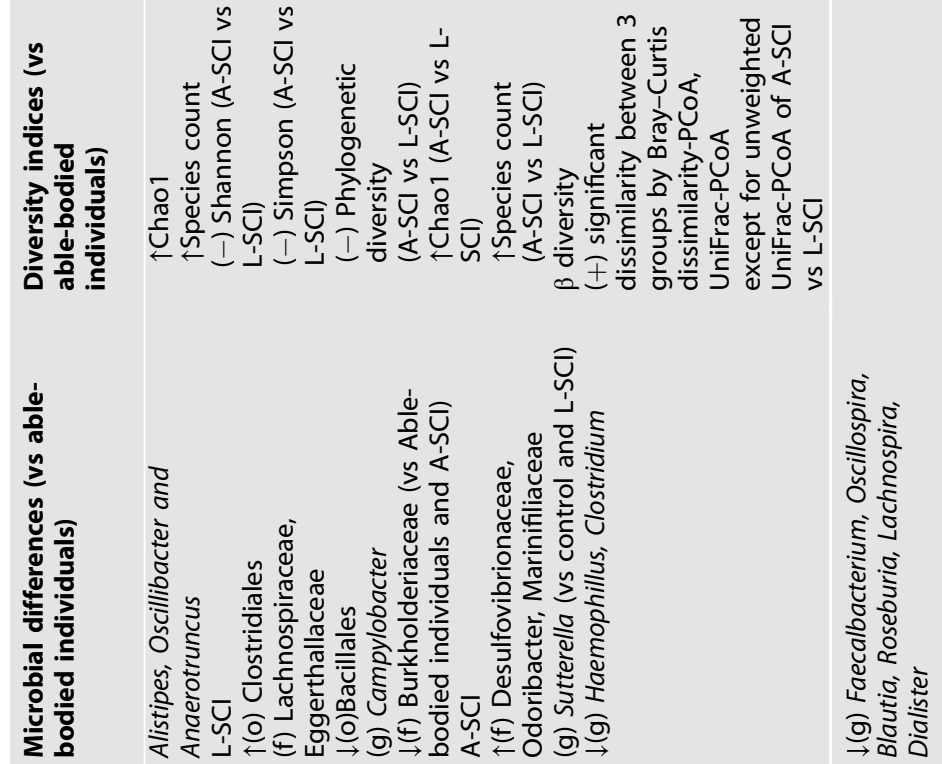

를

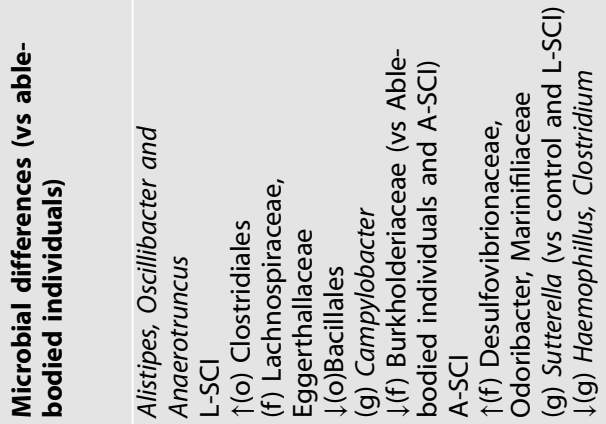

Uे⿺

霅。

흥

은

है वे

흘

ธิ ڤ

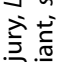

.

흥 은

즘

के 웜.

苟

ডي

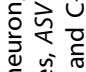

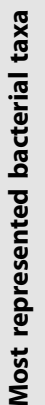
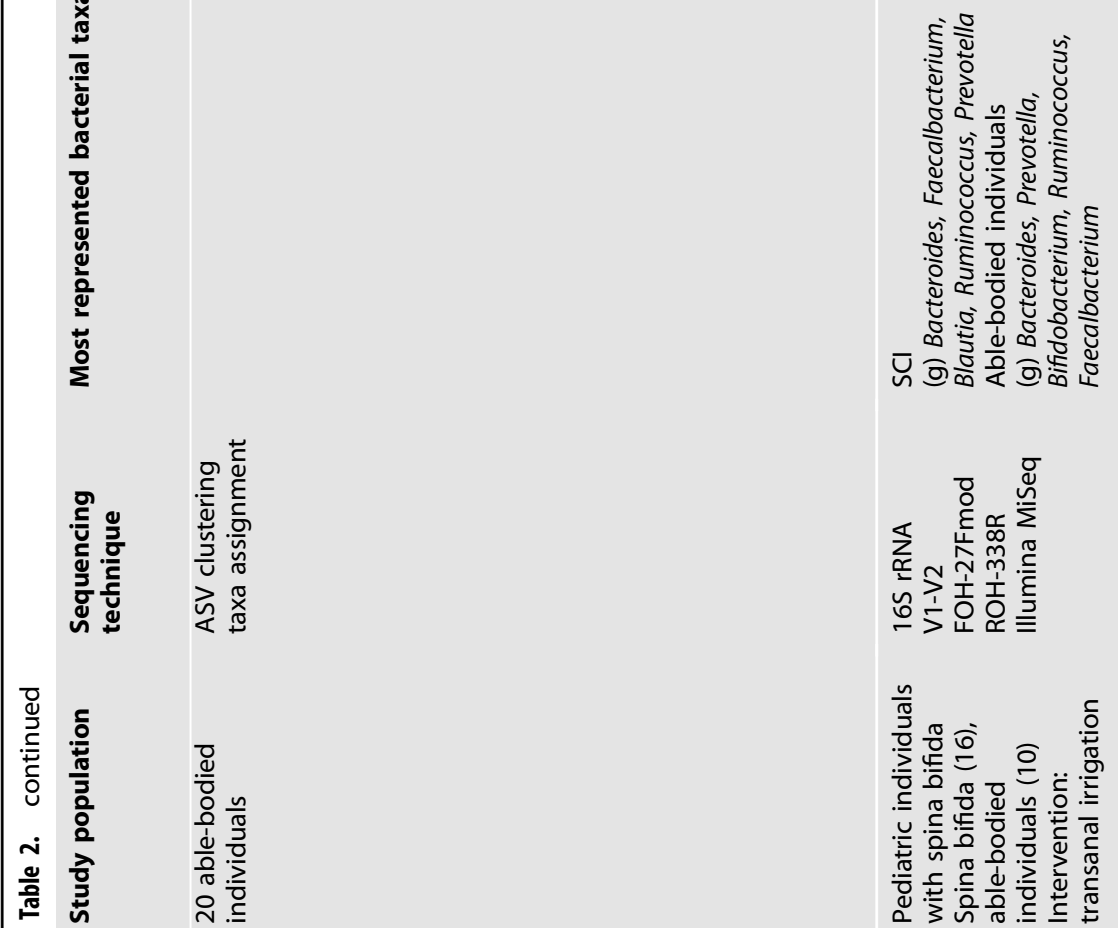

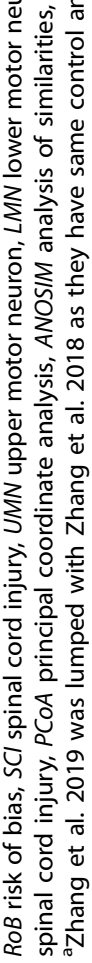


function loss was not described. They found no significant difference in a diversity but $\beta$ diversity was significantly different between individuals with $\mathrm{SCl}$ vs able-bodied individuals. They showed enrichment of Parabacteroides, Alistipes, Phascolactobacterium, Eggerthella, Intestimonas, Flavonifractor, Christensella, Barnesiella, Holdemama, Gordonibacter, Bilophila, and Coprobacillus while depletion of Haemophilus, Clostridium sensu stricto1, Veillonella, Dialister, Roseburia, Megamonas, Subdoligranulum, Leuconostoc, Lachnospira, Megasphaera, Rhodococcus, Ruminococcus, Pseudobutyrivibrio, and Faecalbacterium in individuals with $\mathrm{SCl}$. Li et al. [21], on the other hand, compared patients with acute SCI and those with chronic $\mathrm{SCl}$ to able-bodied individuals. They observed that the a diversity indices were sensitive to species count and were significantly higher among individuals with $\mathrm{SCl}$ and those with acute $\mathrm{SCl}$ had significantly higher diversity compared to the chronic $\mathrm{SCl}$ group. The significant composition differences observed between the groups were bacterial genera changes that are linked to metabolic and neurologic disorders, antibiotic use, and intestinal inflammation.

Furuta et al. [20] investigated the effect of transanal irrigation in improving constipation among pediatric spina bifida individuals' vs healthy able-bodied individuals. Transanal irrigation significantly improved constipation symptoms as measured with Bristol scores and neurogenic bowel dysfunction scores. Faecalbacterium, Oscillospira, Blautia, Roseburia, Lachnospira, Dialister were significantly decreased among individuals with spina bifida and Roseburia correlated positively with the Bristol scores. The use of transanal irrigation significantly increased Bacteroides and Ruminococcus, and decreased Turicibacter.

\section{Spinal cord injury and urinary tract microbiome}

There were six studies on the urinary tract microbiome. Five studies were on adult populations with neurogenic bladder dysfunction secondary to $\mathrm{SCl}$. One study reported a subgroup of children with spina bifida [23] and one was exclusively on children [24]. All studies utilized varying DNA extraction methods but all used the 16S rRNA variable regions for sequencing. All studies showed polymicrobial urine in individuals with $\mathrm{SCl}$. Summarized findings of the microbiome changes are shown in Table 3. All the urine studies had moderate risk of bias. The studies did not account for confounding variables and the extent of injury in the study populations were not sufficiently described [23-28] (Supplementary Table 1).

Three studies had interventions with two of the studies investigating a probiotic treatment. The study of Forster et al. [23] was a phase 1a clinical trial on intravesical instillation of $L$. rhamnosus in children with spina bifida and adults with traumatic $\mathrm{SCl}$. No significant changes in a diversity index between the pre and post-instilation microbiomes were detected, but the microbiome composition between children and adults was significantly different. They observed that the majority of bacteria preinstillation and post-instillation were the same but significantly changed in proportions. On the other hand, Bossa et al. [25] recruited by convenience three individuals from the ProSCIUTTU clinical trial on probiotics containing $L$. rhamnosus with $L$. reuteri and $L$. rhamnosus with Bifidobacterium and observed for their urine catheter flora. They were observed longitudinally for 6 months during the probiotic treatment and for another 2 years thereafter. Two of the patients had no clinically significant UTI while one had a single episode requiring catheter change during the study. Analysis of the terminal restriction fragment polymorphism and the sequences between patients was significantly changed. Among patients, the microbiome was similar pre and posttreatment with probiotics. Their longitudinal analysis showed that the microbiome changed with the onset of probiotics or the presence of symptomatic UTI but reverts to baseline microbiome profile. Philippova et al. [26] studied the effect of intradetrusor botulinum in 6 adults ( 2 males, 4 females) with SCI from trauma or spina bifida. The intervention decreased the occurrence of symptomatic UTI after 6 months of observation. All the urine microbiome prior to intervention was dominated by Enterobacterales but three of the females were shifted to predominantly Lactobacillus urine microbiome. The urine culture result was confirmed by the sequencing data with Escherichia, Klebsiella, Lactobacillus, and Enterococcus as the most common bacterial genera identified.

Fouts et al. [28] included healthy abled body individuals and compared to individuals with $\mathrm{SCl}$ who voided spontaneously (30\%), with intermittent catheterization (30\%), and with indwelling catheters (40\%); females represented $44 \%$ of the population. They found that the healthy able-bodied individuals' urine microbiome was similar up to 2 months after $-\mathrm{SCl}$ but was different in those with 13 months and more after $\mathrm{SCl}$. The presence of Enterococcus and Escherichia in chronic cases significantly contributed to the differences observed. Groah et al. [27] re-analyzed the data using a newer bioinformatics tool and found Escherichia coli and Enterococcus faecalis were significantly enriched along with Pseudomocas aeruginosa and Klebsiella pneumoniae in SCl. They likewise showed that Lactobacillus crispatus was significantly more abundant in female able-bodied individuals vs females with $\mathrm{SCl}$ and Staphylococcus haemolyticus in male able-bodied individuals vs males with $\mathrm{SCl}$. Moreover, they found that members of Enterobacteriaceae were significantly enriched while Lactobacillaceae were significantly depleted among those persons with $\mathrm{SCl}$ using suprapubic catheters and intermittent catheterization compared to able-bodied individuals but not to those persons with $\mathrm{SCl}$ who could void. The diversity indices across all groups and sex, presence of pyuria was not significantly different. There were on average 18 phylotypes (taxon-neutral phylogenic types) that supported the idea of polymicrobial urine.

Forster et al. [24] studied children with neurogenic bladder due to myelomeningocele, anorectal malformation, and tethered cord and who were using intermittent catheterization. They found no significant difference in the a diversity indices and no significant difference in $\beta$ diversity. Members of Enterobacteriaceae was the predominant bacteria identified in those with asymptomatic bacteriuria and UTI while Staphylococcus was the dominant genera among those with negative urine culture after sequencing.

\section{DISCUSSION}

\section{Gut microbiome of individuals with $\mathrm{SCl}$}

Studies on gut microbiomes among individuals with $\mathrm{SCl}$ are limited. They indicate that there is gut dysbiosis post-SCl, verified by changes in the microbiome analysis in both animal studies and human clinical studies. There is a consistent significant $\beta$ diversity difference in gut microbiome composition between individuals with $\mathrm{SCl}$ and able-bodied individuals. The a diversity indices are inconsistent. $\mathrm{SCl}$ seems to induce gut leakiness and bacterial translocation leading to an imbalance in immune response resulting in persistent inflammation. The dominant bacterial genera remain unchanged post- $\mathrm{SCl}$ but butyrateproducing bacteria such as Faecalbacterium, Megamonas, Roseburia are significantly depleted and inflammation-associated bacteria like Alistipes, Anaerotruncus, and Lachnoclostridium are enriched. Interventions such as probiotics, melatonin, and FMT could reverse some of the clinical effects and the dysbiosis associated with $\mathrm{SCl}$.

The gut microbiome of individuals with $\mathrm{SCl}$ is significantly changed with the loss of autonomic nervous system innervation. The loss of control of gut functions and the observed increase in gut permeability allows faster movement of metabolites and gut microbes to cross to blood circulation and induce systemic inflammation [16]. These events change the way the immune system interacts with the gut microorganisms and how they are regulated $[1,3]$, leading to dysbiosis with a consistent decrease 


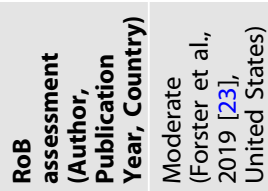

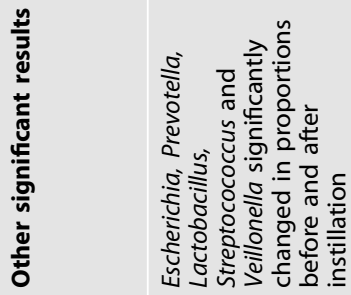

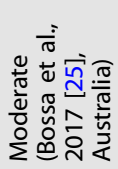

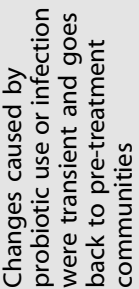

$+x$

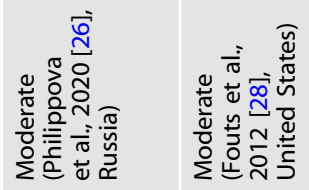

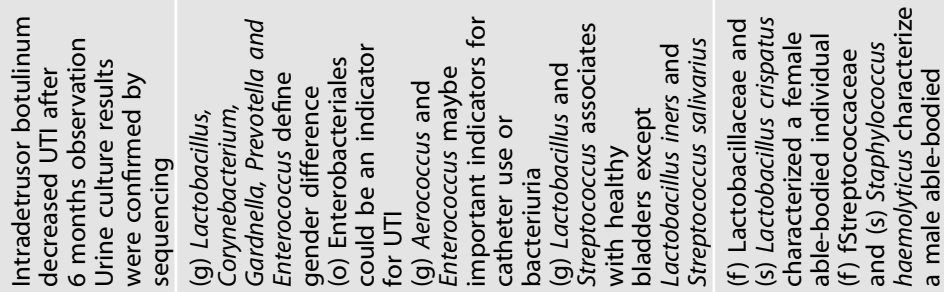

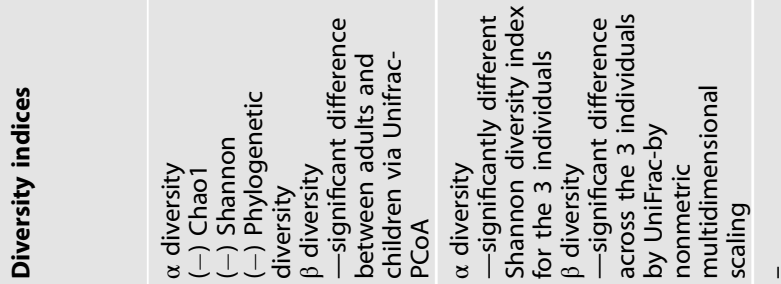

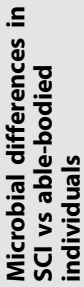

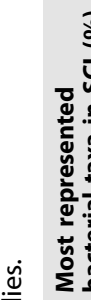

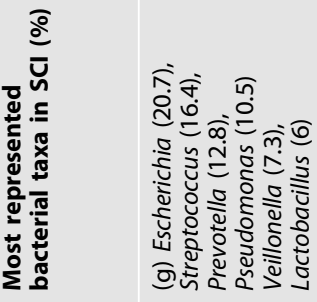

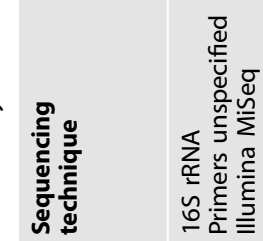

है

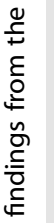

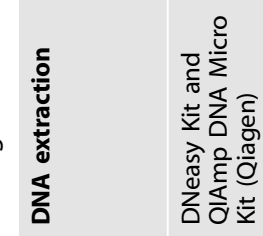

$\mp$

范

है

ले

产

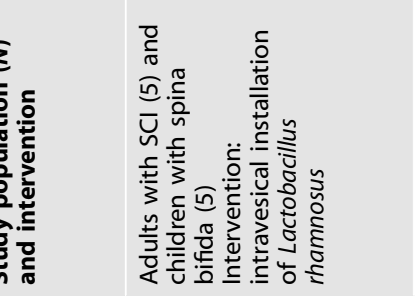

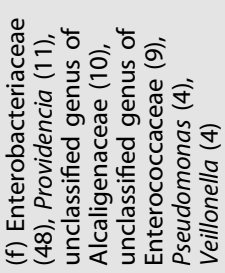

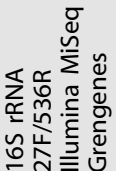

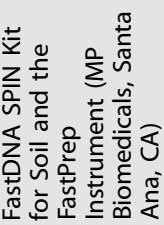

产

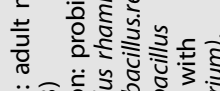

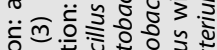

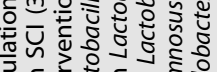
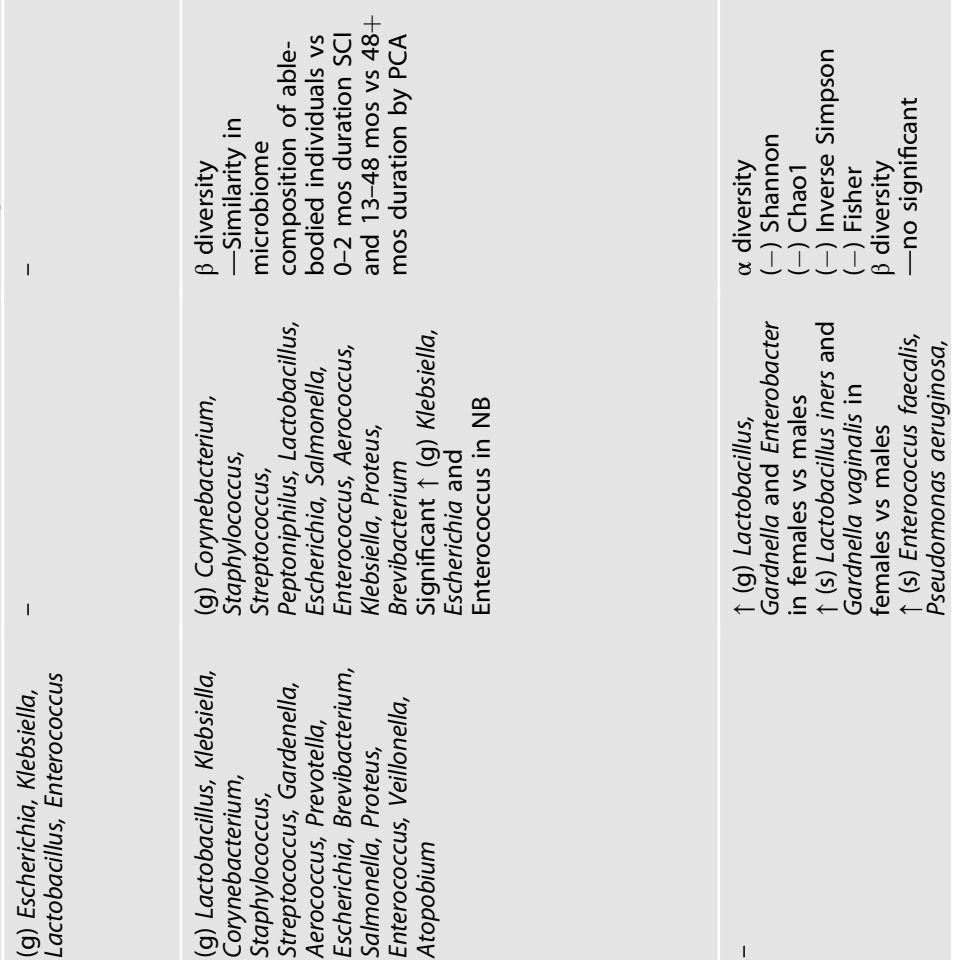

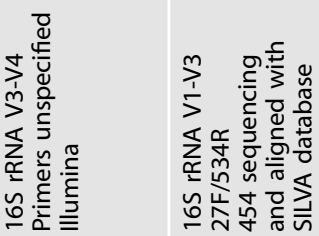
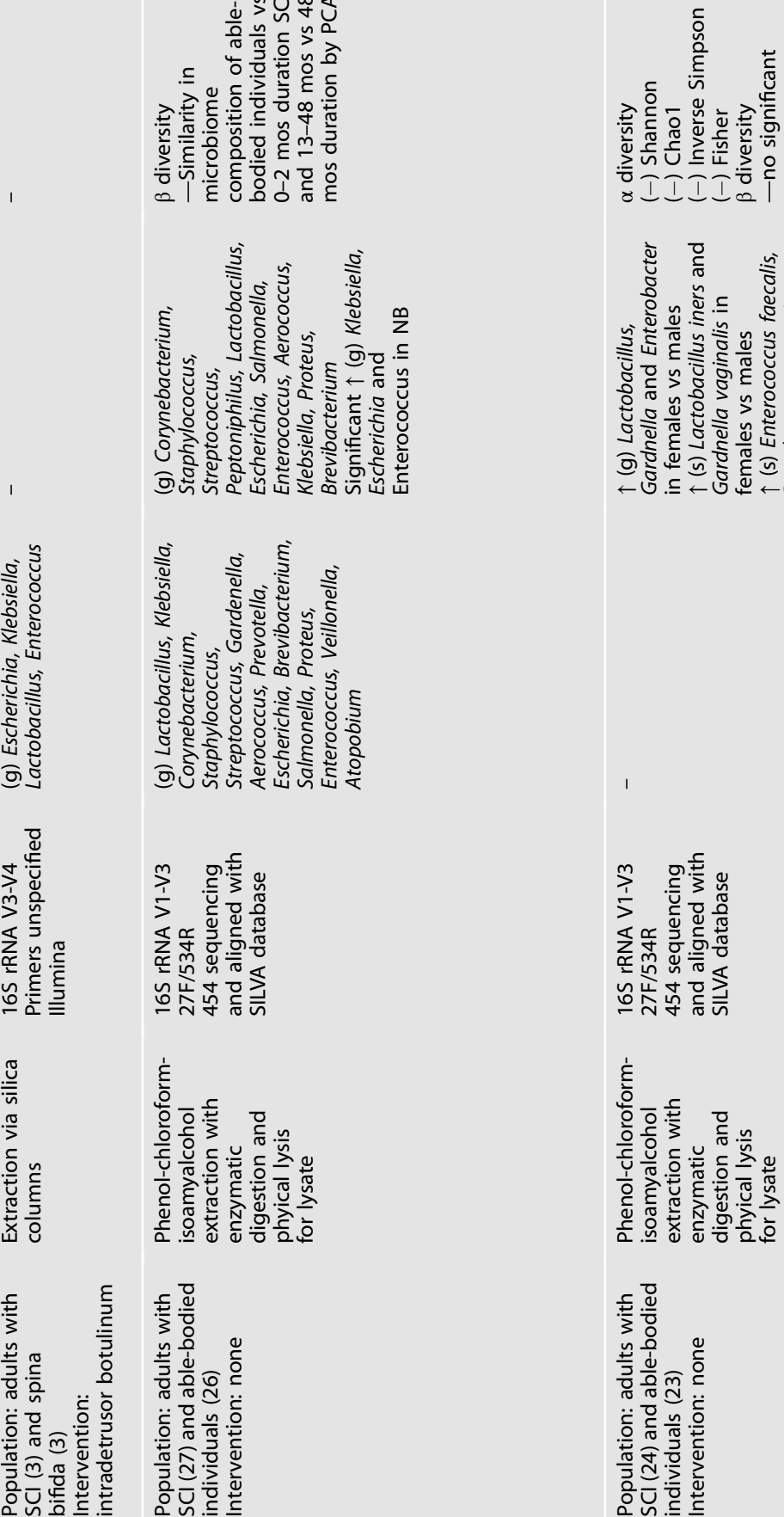

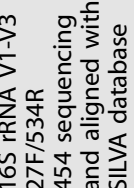

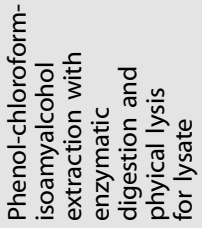

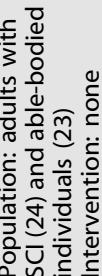




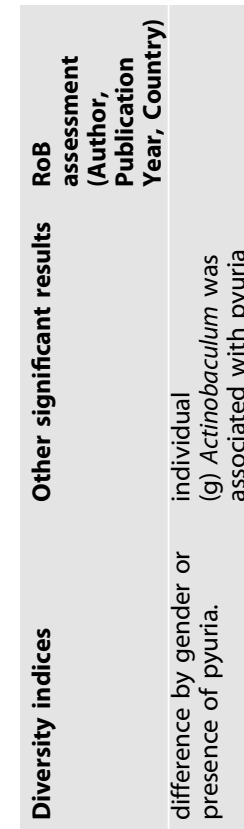

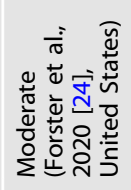

$\frac{0}{0}$
.5
0
0
0
0
$\frac{0}{0}$
$\frac{0}{0}$
.$\frac{1}{2}$
$\frac{0}{0}$
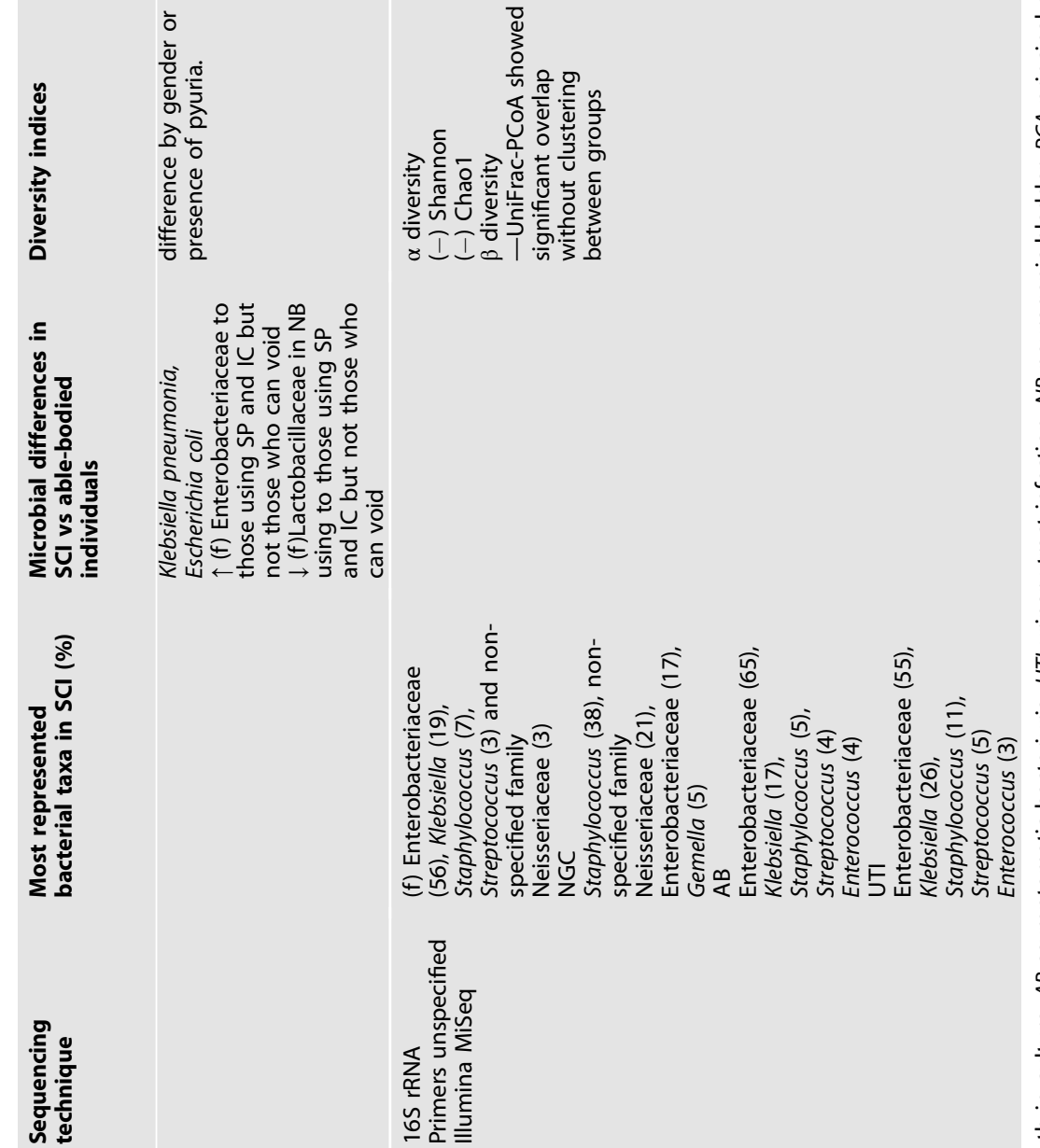

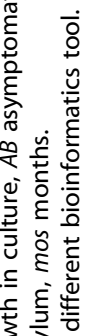
ํํㅇ응

ㄴ. $\frac{5}{5}$

บ흔

지요

产䒽苍

원

过

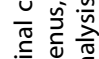

ํㅡㅇ बू

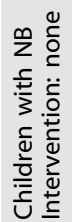

पू बे

包

능

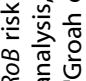


of major butyrate-producing bacteria. Butyrate, a short-chain fatty acid produced by fermentation in the colon, is an energy source for epithelial cells and able to stimulate the production of mucin, antimicrobial peptides, and tight junction proteins which leads to an improved gut mucosal barrier, modulation of the immune system regulation regarding gut microorganisms and reduces oxidative stress in the colon [29]. Similar to $\mathrm{SCl}$, in clinical and preclinical studies of diseases with chronic inflammation such as inflammatory bowel diseases [30, 31], type 2 diabetes [32,33], and atopic dermatitis [34], gut dysbiosis is characterized by depletion of butyrate-producing bacteria. Moreover, enriched bacterial genera such as Alistipes, Anaerotruncus, and Lachnoclostridium are associated with inflammation and obesity [35-41]. Individuals with $\mathrm{SCl}$ are at risk of developing obesity and metabolic disorders [42]. Increasing adiposity among this population is both a consequence of their limited physical activity resulting in positive energy balance but results as well in systemic inflammation associated with chronic $\mathrm{SCl}$ [43]. The gut dysbiosis observed has been likewise linked to anxiety, mood, behavior symptoms, and susceptibility to infection $[1,3,4]$.

The a diversity of the gut microbiome did not significantly differ consistently in $\mathrm{SCl}$ compared to able-bodied individuals. After $\mathrm{SCl}$ a new gut environment could allow new species to proliferate for some time but eventually, these are outgrown by predominant species resulting in a lack of change in species abundance distribution. These observations are time-limited. The more acute the observation, the more a diversity could change significantly. The time of measurement in the animal studies in the review ranged from 3 to 56 days post-SCl but the clinical studies all included individuals with chronic $\mathrm{SCl}$ except for one. This lack of similar time observation could account for the inconsistency observed.

\section{Urinary tract microbiome of individuals with $\mathbf{S C I}$}

There are very few studies on the urinary tract microbiome but the results establish that urine among individuals with $\mathrm{SCl}$ is polymicrobial. Members of Enterobacteriaceae (E. coli, $K$. pneumonia) predominantly populate the urine in $\mathrm{SCl}$ in the review. The use of probiotics and antibiotics induce transient but significant shifts in the urinary tract microbiome. The microbial composition of urine microbiome of individuals with $\mathrm{SCl}$ vs able-bodied individuals is not significantly different regardless of sex. The microbial composition among children's urine is similar in those with UTI, asymptomatic bacteriuria, or negative culture, but significantly differs from the composition in adults.

Urine in individuals with $\mathrm{SCl}$ is polymicrobial and similar to published data on able-bodied individuals there is a similarity in the identified bacterial genera but differs in the dominant group. Lactobacillus and Streptococcus are well represented in ablebodied individuals [44] while members of Enterobacteriaceae such as $E$. coli and $K$. pneumoniae are the dominant bacterial species in the urine of individuals with $\mathrm{SCl}$ especially among those already with UTI and asymptomatic bacteriuria. The dominance of pathogenic members of Enterobacteriaceae could be contributory to the increased risk to UTI, recurrent UTI, and development of chronic kidney failure. Symptomatic UTI and recurrent UTI in this population are frequent and caused mostly by E. coli, K. pneumoniae, P. aeruginosa, E. faecalis, Staphylococcus aureus, and Proteus mirabilis [45]. The difference in the microbiome, however, cannot be fully ascribed to $\mathrm{SCl}$ especially the increase in Enterobacteriaceae is seen mostly among those who use suprapubic catheters and intermittent catheters but not among those who can void when compared to able-bodied individuals [27]. This raises the possibility that catheter use influences the urinary microbiome as well in the $\mathrm{SCl}$ population. Moreover, studies in this review show that there is similar composition of bacterial genera among those with no UTI and those with UTI. There is simply a change in the dominant bacteria when there is UTI. This could explain the observed effect of probiotics and antibiotics which lead to significant but transient changes in the urinary microbiome but the microbiome revert to pre-existing composition after use [25].

\section{Strengths and limitations of current study and directions for future research}

The review summarized only studies with genomic sequencing microbiome results. The use of the collective genome allowed the studies to identify organisms previously unidentifiable and even nonviable organisms in culture. This provides better resolution on which bacteria are present in the body sites. The method has limitations especially regarding collection methods, sequencing technique and bioinformatics tool use could give different results. Low biomass samples such as from urine and skin are prone to contamination and host DNA interference. Different sequencing technique has different DNA quality needs, some process only a specific DNA length, introduce bias when amplification is use and has different error rates for reading nucleotide bases. Bioinformatics tools, likewise, use different reference databases and some have only up to a limited level of taxonomic classification.

The search strategy for the review was broad to accommodate terms for multiple body sites and tried to capture early studies as the indexing term microbiota and microbiome are recent. The studies in the review are mostly observational and provide snapshots of a dynamic process in the gut and the urinary tract. They provide limited information and do not capture the dynamic interactions of the organisms. Likewise, microbiomes are affected by age, sex, diet, physical activity, comorbidities, level of injury, duration of injury, and use of therapeutics [1]. Therefore, future studies would benefit from controlling for more variables directly affecting the microbiome, varying sample locations, and additional time points for observation. Improvement of the sequencing technologies and reduced costs for whole genome sequencing will allow the microbial characterization at the species level. This is important for future studies wherein a single species might drive the change at the genus level or higher taxa. Studies should focus on microbiomes of organ-systems commonly affected by the loss of innervation after $\mathrm{SCl}$ particularly the urogenital, gut, and skin that may be contributory to the acute and chronic morbidities seen in this group. Clinical studies on immunology and inflammation are needed in persons with $\mathrm{SCl}$ to test whether immune defenses react to changes in the microbiome and why a large number of pathogens especially in the urinary tract are tolerated, as seen in the review.

\section{CONCLUSION}

Studies on microbiomes in $\mathrm{SCl}$ remain limited to the gut and the urinary tract. Dysbiosis post-SCl is present and the microbiome profiles follow patterns associated with diseases with chronic inflammation and metabolic disorders. The urinary tract, on the other hand, shows a microbiome that shifts in composition post$\mathrm{SCl}$ with the dominance of UTI-associated organisms. Dynamic changes in the microbiome can be used clinically as a specific target for therapeutics to correct dysbiosis or to reduce the risk of disease.

\section{DATA AVAILABILITY}

All data generated or analyzed during the current study are included in this published article and its Supplementary information files. 


\section{REFERENCES}

1. Kigerl KA, Zane K, Adams K, Sullivan MB, Popovich PG. The spinal cord-gutimmune axis as a master regulator of health and neurological function after spinal cord injury. Exp Neurol. 2020;323:113085.

2. Gill SR, Pop M, DeBoy RT, Eckburg PB, Turnbaugh PJ, Samuel BS, et al. Metagenomic analysis of the human distal gut microbiome. Science. 2006;312:1355-9.

3. Jogia T, Ruitenberg MJ. Traumatic spinal cord injury and the gut microbiota: current insights and future challenges. Front Immunol. 2020;11:704.

4. Wallace DJ, Sayre NL, Patterson TT, Nicholson SE, Hilton D, Grandhi R. Spinal cord injury and the human microbiome: beyond the brain-gut axis. Neurosurg Focus. 2019;46:E11.

5. Moher D, Liberati A, Tetzlaff J, Altman DG, Prisma Group. Reprint-preferred reporting items for systematic reviews and meta-analyses: the PRISMA statement. Phys Ther. 2009;89:873-80.

6. Muka T, Glisic M, Milic J, Verhoog S, Bohlius J, Bramer W, et al. A 24-step guide on how to design, conduct, and successfully publish a systematic review and metaanalysis in medical research. Eur J Epidemiol. 2020;35:49-60.

7. Turnbaugh PJ, Ley RE, Hamady M, Fraser-Liggett CM, Knight R, Gordon J. A strategy to understand the microbial components of the human genetic and metabolic landscape and how they contribute to normal physiology and predisposition to disease. Nature. 2007;449:804-10.

8. Consortium THMP. Structure, function and diversity of the healthy human microbiome. Nature. 2012;486:207-14.

9. National Toxicology Program. OHAT Risk of Bias Rating Tool for Human and Animal Studies. US Department of Health and Human Sciences. 2015. https://ntp. niehs.nih.gov/ntp/ohat/pubs/riskofbiastool_508.pdf. Accessed on 01 Oct 2020.

10. O'Connor G, Jeffrey E, Madorma D, Marcillo A, Abreu MT, Deo SK, et al. Investigation of microbiota alterations and intestinal inflammation post-spinal cord injury in rat model. J Neurotrauma. 2018;35:2159-66.

11. Jing $Y, Y u Y$, Bai $F$, Wang $L$, Yang $D$, Zhang $C$, et al. Effect of fecal microbiota transplantation on neurological restoration in a spinal cord injury mouse model: involvement of brain-gut axis. Microbiome. 2021;9:1-21.

12. Jing $Y$, Yang D, Bai F, Zhang C, Qin C, Li D, et al. Melatonin treatment alleviates spinal cord injury-induced gut dysbiosis in mice. J Neurotrauma. 2019;36:2646-64.

13. Schmidt EKA, Torres-Espin A, Raposo PJF, Madsen KL, Kigerl KA, Popovich PG, et al. Fecal transplant prevents gut dysbiosis and anxiety-like behaviour after spinal cord injury in rats. PLoS ONE. 2020;15:e0226128.

14. Schmidt EKA, Raposo PJF, Madsen KL, Fenrich KK, Kabarchuk G, Fouad K. What Makes a Successful Donor? Fecal Transplant from Anxious-Like Rats Does Not Prevent Spinal Cord Injury-Induced Dysbiosis. Biology. 2021;10:24.

15. Myers SA, Gobejishvili L, Saraswat Ohri S, Garrett Wilson C, Andres KR, Riegler AS, et al. Following spinal cord injury, PDE4B drives an acute, local inflammatory response and a chronic, systemic response exacerbated by gut dysbiosis and endotoxemia. Neurobiol Dis. 2019;124:353-63.

16. Kigerl KA, Hall JC, Wang L, Mo X, Yu Z, Popovich PG. Gut dysbiosis impairs recovery after spinal cord injury. J Exp Med. 2016;213:2603-20.

17. Zhang $C$, Jing $Y$, Zhang W, Zhang J, Yang $M$, Du $L$, et al. Dysbiosis of gut microbiota is associated with serum lipid profiles in male patients with chronic traumatic cervical spinal cord injury. Am J Transl Res. 2019;11:4817-34.

18. Zhang $C$, Zhang W, Zhang J, Jing Y, Yang M, Du L, et al. Gut microbiota dysbiosis in male patients with chronic traumatic complete spinal cord injury. J Transl Med. 2018:16:353.

19. Lin R, Xu J, Ma Q, Chen M, Wang L, Wen S, et al. Alterations in the fecal microbiota of patients with spinal cord injury. PLoS ONE. 2020;15:e0236470.

20. Furuta A, Suzuki Y, Takahashi R, Jakobsen BP, Kimura T, Egawa S, et al. Effects of transanal irrigation on gut microbiota in pediatric patients with spina bifida. J Clin Med. 2021;10:1-12.

21. Li J, Van Der Pol W, Eraslan M, McLain A, Cetin H, Cetin B, et al. Comparison of the gut microbiome composition among individuals with acute or long-standing spinal cord injury vs. able-bodied controls. J Spinal Cord Med. 2020;4:1-9.

22. Gungor B, Adiguzel E, Gursel I, Yilmaz B, Gursel M. Intestinal microbiota in patients with spinal cord injury. PLoS ONE. 2016;11:e0145878.

23. Forster CS, Hsieh MH, Perez-Losada M, Caldovic L, Pohl H, Ljungberg I, et al. A single intravesical instillation of Lactobacillus rhamnosus GG is safe in children and adults with neuropathic bladder: A phase la clinical trial. J Spinal Cord Med. 2019;44:1-8.

24. Forster CS, Panchapakesan K, Stroud C, Banerjee P, Gordish-Dressman H, Hsieh MH. A cross-sectional analysis of the urine microbiome of children with neuropathic bladders. J Pediatr Urol. 2020;16:593.e1-593.e8. https://doi.org/ 10.1016/j.jpurol.2020.02.005

25. Bossa L, Kline K, McDougald D, Lee BB, Rice SA. Urinary catheter-associated microbiota change in accordance with treatment and infection status. PLOS ONE. 2017. https://doi.org/10.1371/journal.pone.0177633.

26. Philippova ES, Bazhenov IV, Zyrianov AV. Does botulinum therapy affect the urine microbiome of patients with neurogenic bladder? Metagenomic sequencing results. Vestn Urol. 2020;8:85-96.
27. Groah SL, Perez-Losada M, Caldovic L, Ljungberg IH, Sprague BM, Castro-Nallar E, et al. Redefining Healthy Urine: A Cross-Sectional Exploratory Metagenomic Study of People With and Without Bladder Dysfunction. J Urol. 2016;196:579-87.

28. Fouts DE, Pieper R, Szpakowski S, Pohl H, Knoblach S, Suh MJ, et al. Integrated next-generation sequencing of $16 \mathrm{~S}$ rDNA and metaproteomics differentiate the healthy urine microbiome from asymptomatic bacteriuria in neuropathic bladder associated with spinal cord injury. J Transl Med. 2012;10:174.

29. Hamer HM, Jonkers D, Venema K, Vanhoutvin S, Troost FJ, Brummer RJ. Review article: the role of butyrate on colonic function. Aliment Pharm Ther. 2008; 27:104-19.

30. Machiels K, Joossens M, Sabino J, De Preter V, Arijs I, Eeckhaut V, et al. A decrease of the butyrate-producing speciesRoseburia hominisandFaecalibacterium prausnitziidefines dysbiosis in patients with ulcerative colitis. Gut. 2014;63 $1275-83$.

31. Parada Venegas D, De la Fuente MK, Landskron G, Gonzalez MJ, Quera R, Dijkstra G, et al. Short Chain Fatty Acids (SCFAs)-Mediated Gut Epithelial and Immune Regulation and Its Relevance for Inflammatory Bowel Diseases. Front Immunol. 2019;10:277.

32. Qin J, Li Y, Cai Z, Li S, Zhu J, Zhang F, et al. A metagenome-wide association study of gut microbiota in type 2 diabetes. Nature. 2012;490:55-60.

33. Delzenne NM, Cani PD, Everard A, Neyrinck AM, Bindels LB. Gut microorganisms as promising targets for the management of type 2 diabetes. Diabetologia. 2015;58:2206-17.

34. Nylund L, Nermes M, Isolauri E, Salminen S, de Vos WM, Satokari R. Severity of atopic disease inversely correlates with intestinal microbiota diversity and butyrate-producing bacteria. Allergy. 2015;70:241-4.

35. Andoh A, Nishida A, Takahashi K, Inatomi O, Imaeda $H$, Bamba $S$, et al. Comparison of the gut microbial community between obese and lean peoples using $16 \mathrm{~S}$ gene sequencing in a Japanese population. J Clin Biochem Nutr. 2016;59:15-152.

36. Xu P, Wang J, Hong F, Wang S, Jin X, Xue T, et al. Melatonin prevents obesity through modulation of gut microbiota in mice. J Pineal Res. 2017. https://doi.org/ 10.1111/jpi.12399.

37. Kong C, Gao R, Yan X, Huang L, Qin H. Probiotics improve gut microbiota dysbiosis in obese mice fed a high-fat or high-sucrose diet. Nutrition. 2019;60:175-84.

38. Kaplan RC, Wang Z, Usyk M, Sotres-Alvarez D, Daviglus ML, Schneiderman N, et al. Gut microbiome composition in the Hispanic Community Health Study/Study of Latinos is shaped by geographic relocation, environmental factors, and obesity. Genome Biol. 2019. https://doi.org/10.1186/s13059-019-1831-z.

39. Zhao L, Zhang Q, Ma W, Tian F, Shen $H$, Zhou M. A combination of quercetin and resveratrol reduces obesity in high-fat diet-fed rats by modulation of gut microbiota. Food Funct. 2017;8:4644-56.

40. Wang $P$, Li D, Ke W, Liang D, Hu X, Chen F. Resveratrol-induced gut microbiota reduces obesity in high-fat diet-fed mice. Int J Obes. 2019;44:213-25.

41. Zhou L, Ni Z, Yu J, Cheng W, Cai Z, Yu C Correlation Between Fecal Metabolomics and Gut Microbiota in Obesity and Polycystic Ovary Syndrome. Front Endocrinol 2020. https://doi.org/10.3389/fendo.2020.00628.

42. Gorgey AS, Gater DR Jr. Prevalence of obesity after spinal cord injury. Top Spinal Cord Inj Rehabil. 2007;12:1-7.

43. Farkas GJ, Gater DR. Neurogenic obesity and systemic inflammation following spinal cord injury: a review. J Spinal Cord Med. 2017;41:378-87.

44. Aragon IM, Herrera-Imbroda B, Queipo-Ortuno MI, Castillo E, Del Moral JS, Gomez-Millan J, et al. The urinary tract microbiome in health and disease. Eur Urol Focus. 2018;4:128-38.

45. Garcia-Arguello LY, O'Horo JC, Farrell A, Blakney R, Sohail MR, Evans CT, et al. Infections in the spinal cord-injured population: a systematic review. Spinal Cord. 2017;55:526-34.

\section{AUTHOR CONTRIBUTIONS}

Study concept and design: MG and JS; search strategy creation and online database search: DKH; acquisition, collection, interpretation of data: $E V, T P, A B, O I, G F, J P, M G$, JS; drafting of the manuscript: $E V, M G, J S$; critical revision of the manuscript for important intellectual content: EV, AB, TP, OI, GF, DKH, JP, MG, JS. Study supervision: MG and JS; All authors approved the final version of the manuscript.

\section{FUNDING}

This study is partially funded by the Swiss Paraplegic Foundation. The project has received funding also from the European Union's Horizon 2020 research and innovation program under the Marie Skłodowska-Curie grant agreement No 801076, through the SSPH+Global PhD Fellowship Program in Public Health Sciences (GlobalP3HS) of the Swiss School of Public Health. 


\section{COMPETING INTERESTS}

The authors declare no competing interests.

\section{ADDITIONAL INFORMATION}

Supplementary information The online version contains supplementary material available at https://doi.org/10.1038/s41393-021-00737-y.

Correspondence and requests for materials should be addressed to Ezra Valido.

Reprints and permission information is available at http://www.nature.com/ reprints

Publisher's note Springer Nature remains neutral with regard to jurisdictional claims in published maps and institutional affiliations.
cC (i) Open Access This article is licensed under a Creative Commons Attribution 4.0 International License, which permits use, sharing, adaptation, distribution and reproduction in any medium or format, as long as you give appropriate credit to the original author(s) and the source, provide a link to the Creative Commons license, and indicate if changes were made. The images or other third party material in this article are included in the article's Creative Commons license, unless indicated otherwise in a credit line to the material. If material is not included in the article's Creative Commons license and your intended use is not permitted by statutory regulation or exceeds the permitted use, you will need to obtain permission directly from the copyright holder. To view a copy of this license, visit http://creativecommons. org/licenses/by/4.0/.

(c) The Author(s) 2022 